\title{
quelques développements récents dans le calcul dynamique des barrages en terre
}

\author{
some recent developments in dynamic design of earth dams
}

\author{
T. AVRIL \\ Ingénieur EDF à la région Equipement Alpes-Lyon *
}

Rev. Franç. Géotech. n 46, p.p. 9-18 (janvier 1989)

\section{Résumé}

Cet article fait le point sur les méthodes de calcul dynamique des barrages en terre pratiquées à Electricité de France. Les analyses simplifiées et les méthodes aux éléments finis présentent chacune leurs avantages et leurs limites d'application. On montre qu'il est aujourd'hui possible d'évaluer le comportement irréversible de l'ouvrage à partir de lois de comportement non linéaire. La détermination des paramètres dynamiques en laboratoire et in situ pose des problèmes spécifiques à ce type d'ouvrage, dont on présente quelques exemples d'application. Enfin pour les calculs à la rupture, et la génération de pressions interstitielles en fin de séisme, la pratique consiste à utiliser des approches simplifiées à partir de la réponse en contraintes totales de l'ouvrage.

\section{Abstract}

This paper summarizes the earth dam dynamic methods which are practised at Electricité de France. Simplified, and finite element methods have both advantages and limit of applicability. An example is shown of dam irreversible response based on now available nonlinear strain-stress laws for soils. Laboratory or in situ dynamic characteristics determination present difficulties in relation to this type of structure. Some pratical examples are shown. At last, for stability calculations and generation of pore water pressures, simplified procedures are used based on the dynamic total stress response of the dam.

- 3 et 5 , rue Ronde, 73010 Chambéry. 


\section{NOTATIONS}

$G_{\max } \quad$ Module de cisaillement aux faibles déformations $(\mathrm{MPa})$

e Indices des vides $(-)$

$\mathrm{p} \quad$ Pression de confinement (MPa)

$\mathrm{p}_{\text {ră }} \quad$ Pression de référence

\section{INTRODUCTION}

L'observation pendant les récents séismes du comportement des barrages en terre montre que ces ouvrages peuvent supporter des secousses très importantes lorsqu'ils sont bien construits (réf. 31, 19). En réalité ce n'est pas tant le niveau de sollicitation qui est significatif, mais la nature et l'état de compacité des matériaux qui sont capitaux. Les barrages qui se sont rompus étaient, soit fondés sur un sol de qualité médiocre (SHEFFIELD, 1925), soit mis en place sans précaution particulière, le plus souvent par remblayage hydraulique (SAN FERNANDO, 1971). La cause principale de rupture est la perte de résistance au cisaillement des maté. riaux, mais d'autres désordres peuvent entraîner un disfonctionnement de l'ouvrage: les tassements irréversibles de plusieurs dizaines de centimètres, qui entraînent une perte de revanche et qui peuvent s'aggraver lors de séismes successifs ; ou bien les ouvertures de fissures le plus souvent longitudinales, parfois transversales, qui augmentent considérablement le risque de rupture par érosion interne. De nombreux moyens d'analyse sont aujourd'hui disponibles pour étudier ces problèmes; cependant ils sont parfois difficiles à mettre en cuure parce que parallèlement notre connaissance du comportement dynamique des sols reste relativement en retard. De nombreuses études en cours, en particulier dans le cadre du GRECO "Rhéologie des Géomatériaux », cherchent d'une part à préciser le domaine d'application des différentes lois de comportement sous chargement dynamique, d'autre part à développer les essais de laboratoire et les essais in situ capables de fournir les paramètres de comportement de chaque modèle. Il revient au projecteur de veiller dans chaque cas à adapter le type de calcul en fonction de la connaissance qu'il a du a, $\mathrm{n}$ Constantes caractéristiques des matériaux (-)

$\left(\lambda_{1}, \mu_{3}\right)$ Coefficients de Lamé à la base du barrage (MPa)

(o) Masse volumique des matériaux $\left(\mathrm{kg} / \mathrm{m}^{3}\right)$

$t_{1} \quad$ Pente de talus amont

$t_{2} \quad$ Pente de talus aval

$\mathrm{n}_{1} \quad$ Pente du noyau à lamont

$\mathrm{n}_{2} \quad$ Pente du noyau à l'aval

comportement dynamique des matériaux, et de vérifier en fin d'analyse que les hypothèses de départ ont bien été respectées.

\section{COMPORTEMENT DYNAMIQUE DES BARRAGES}

\subsection{Sollicitations de faibles amplitudes}

Les essais de mise en vibration forcée de barrages en terre au moyen d'excitateurs mécaniques ont montré que pour les très faibles niveaux de sollicitation, le comportement de l'ouvrage est visco-élastique avec un pourcentage d'amortissement critique compris entre 5 et $10 \%$ (réf. 30). Les essais de laboratoire confirment ce résultat, on trouve selon les matériaux que les propriétés visco-élastiques restent constantes pour des niveaux de déformation de cisaillement inférieurs à $10^{-5}$ ou $10^{-6}$. Les modèles à base de visco-élasticité furent tout naturellement développés en premier : MONONOBE et al. (1936) (réf. 25). Le barrage était supposé homogène, tant du point de vue de la nature que des propriétés des matériaux constitutifs. Il n'est pas difficile de généraliser ce modèle au barrage zoné représenté à la figure 1 , la possibilité de différencier entre trois types de matériaux facilite lutilisation de la méthode. Enfin les propriétés élastiques varient à l'intérieur du barrage en fonction de la hauteur de terre au-dessus du point considéré, c'est-à-dire plus précisément en fonction de la pression de confinement (HARDIN-RICHART, 1963) :

$$
G_{\max }=G_{\text {ref }}\left(\frac{a-e}{1+e}\right)^{2}\left(\frac{p}{p_{\text {reff }}}\right)^{n}
$$

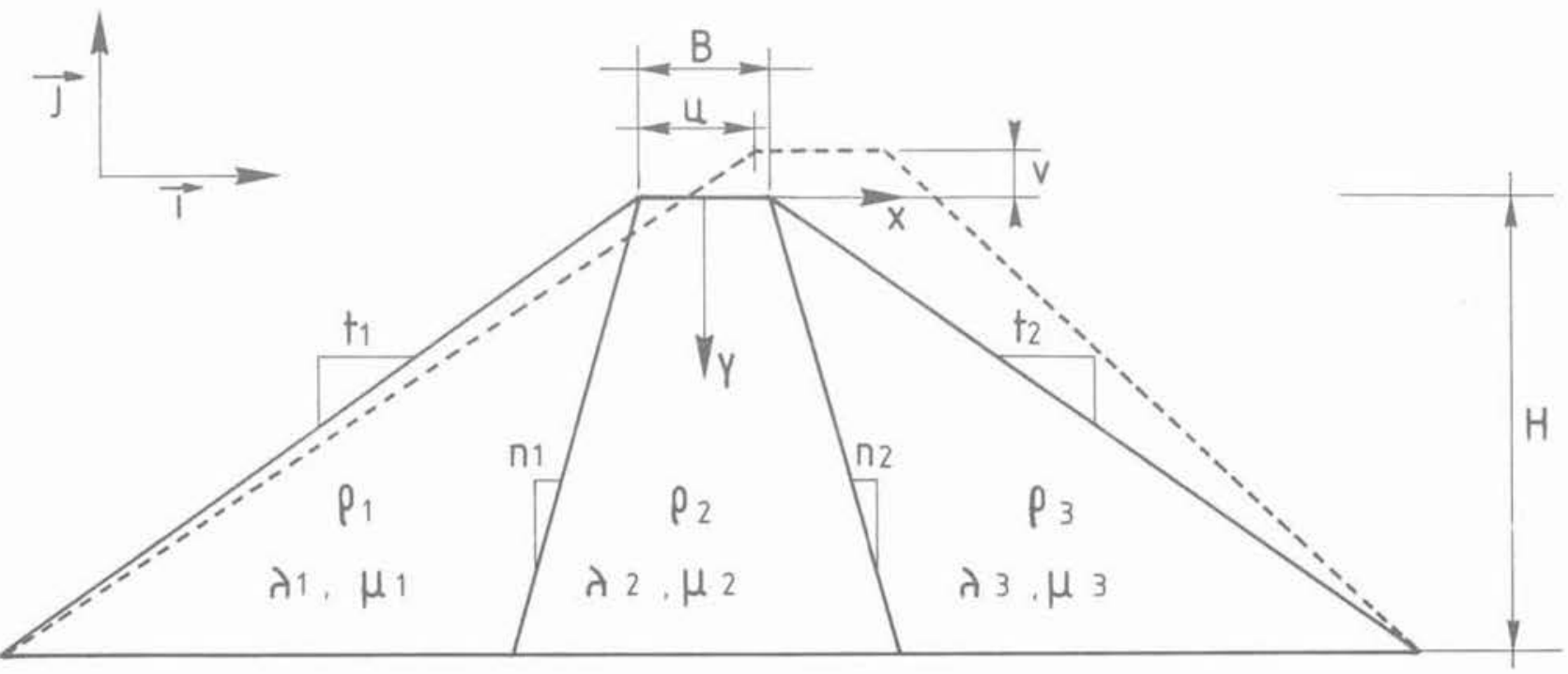

Fig. 1. - Méthode simplifiée - Barrage type.

Fig. 1. - Simplified method - Typical dam. 
La fréquence fondamentale $f_{0}$ du barrage, que l'on peut déterminer à partir des relations (2) à (4), permet d'évaluer les accélérations maximales à différentes hauteurs de l'ouvrage, ainsi que le niveau de déformation de cisaillement atteint (réf. 22).

$$
\begin{aligned}
f_{0}=\frac{1}{2 \pi} \sqrt{\frac{K}{M}} \\
k=\frac{1}{(n+1)(n+2)}\left(\left(\lambda_{1}+2 \mu_{1}\right) t_{1}\left(1-n_{1} / t_{1}\right)^{n+1}\right. \\
+\left(\lambda_{2}+2 \mu_{2}\right) t_{1}\left(1-\left(1-n_{1} / t_{1}\right)^{n+1}\right) \\
+\left(\lambda_{2}+2 \mu_{2}\right)(n+2) B / H \\
+\left(\lambda_{2}+2 \mu_{2}\right) t_{2}\left(1-\left(1-n_{2} / t_{2}\right)^{n+1}\right) \\
\left.+\left(\lambda_{3}+2 \mu_{3}\right) t_{2}\left(1-n_{2} / t_{2}\right)^{n+1}\right) \\
m=\frac{H^{2}}{12}\left(P_{1}\left(t_{1}-n_{1}\right)+P_{2} /\left(4 B / H+n_{1}+n_{2}\right)\right. \\
\left.+P_{3}\left(t_{2}-n_{2}\right)\right)
\end{aligned}
$$

Les valeurs des coefficients de Lamé $\lambda$ et $\mu$, sont à considérer à la base du barrage. Ce point est important, et distingue cette méthode simplifiée de celles proposées auparavant. Dans ces dernières, il n'était pas facile de connaître à quelle hauteur sous la crête du barrage, choisir la valeur représentative des propriétés élastiques de louvrage (réf. 22 , 34).

Les paramètres du modèle peuvent s'obtenir à partir de mesures in situ, soit par des essais cross-hole, soit par des essais de sismique transparence. Les figures 2 et 3 donnent le principe des mesures, et quelques résultats obtenus sur le barrage de Grand'Maison. Ces essais sont d'autant plus intéressants que les granulométries des matériaux mis en place sont en général incompatibles avec la taille des plus gros appareillages de laboratoire. On doit alors couper la granulométrie pour réaliser les essais de laboratoires qui sous-estiment ainsi généralement les valeurs de module de cisaillement par rapport à ceux que Pon peut mesurer in situ (réf. 4).

\subsection{Influence du niveau de déformation}

Quelques rares essais in situ, mais de nombreux essais de laboratoire montrent qu'entre $10^{-5}$ et $10^{-3}$ de déformation de cisaillement le comportement reste globalement visco-élastique, mais que ses propriétés sont fonction du niveau de déformation (figure 4). Les modèles simplifiés précédents peuvent alors être complétés par un processus itératif. A chaque étape, les caractéristiques visco-élastiques des matériaux sont ajustées en fonction du niveau de sollicitation : ceci constitue le principe de la méthode équivalente linéaire. Ce n'est que très récemment que nous avons pu disposer d'un instrument de mesures en laboratoire capable de déterminer l'ensemble des caractéristiques de ce modèle (réf. 10). En ce qui concerne les essais in situ, quelques résultats ont été obtenus, mais avec la mise en œuvre de moyens très importants, incompatibles avec un essai industriel. Dans les barrages en terre, l'état de contrainte et l'état de saturation qui diffèrent d'un point à un autre de l'ouvrage influencent, d'une part, les caractéristiques équivalentes-linéaires, et d'autre part le type de réponse du matériau qui peut devenir non linéaire (réf. 20). On comprend dès lors l'importance et l'intérêt du travail entrepris dans le cadre du GRECO « Rhéologie des Géomatériaux » d'une part sur la détermination in situ des paramètres, et d'autre part sur le domaine de validité de chaque loi de comportement sous chargement cyclique.

\subsection{Comportements non linéaires}

Bien entendu, aucun des modèles précédents n'est capable de prévoir une déformation irréversible, ou même d'estimer correctement les variations de volume des matériaux pendant le chargement dynamique. Si beaucoup de lois de comportement incrémentales ou élastoplastiques ont été proposées, la comparaison avec l'expérience est restée longtemps insuffisante (réf. 28). Les tentatives plus récentes à partir de la loi élastoplastique proposée par J.-C. HUJEUX ont donné des résultats encourageants. On a indiqué sur la figure 4, la courbe équivalente linéaire déduite à partir de la simulation d'un essai triaxial cyclique en condition non drainé sur du sable d'Hostun, et qui se compare bien aux résultats obtenus en laboratoire. L'étape suivante consiste à tester la loi de comportement, soit sur d'autres trajets de chargement à partir du même jeu de paramètre, soit sur un ouvrage réel. Sur la figure 5 , on a indiqué les premiers résultats d'un calcul dynamique monophasique non linéaire de barrage à partir de la loi élastoplastique proposée par J.-C. HUJEUX. II reste encore beaucoup de travail pour valider ce type de modèle, mais la détermination des tassements après séisme, des pressions interstitielles, et des variations de volume à l'intérieur de louvrage est à ce prix. Ces dernières sont à l'origine des pertes de résistance au cisaillement qui ont causé la plupart des ruptures de barrage en terre. Dans le cas de matériaux láches et saturés, un niveau de contrainte cyclique très faible peut conduire à la rupture par laccumulation du nombre de cycle. Ce phénomène de liquéfaction a pu être modélisé par un calcul avec couplage entre le squelette solide et le liquide interstitiel (réf. 24). Cependant ces analyses restent très lourdes d'utilisation, et le projeteur recherche parfois des méthodes moins rigoureuses mais plus faciles d'emploi. Il est intéressant de noter à ce titre que plusieurs modèles ont été proposés pour étudier de manière simplifiée la réponse non linéaire de barrage (réf. 1, 11, 12, 13, 14). Cette voie est certainement très intéressante dans tous les cas où l'on peut se contenter des ordres de grandeur, par exemple au niveau d'une étude d'avant-projet.

\section{PROBLÈMES DE MODÉLISATION}

Les choix de la modélisation et des conditions aux limites du problème sont très importants dans le cas d'un calcul dynamique. S'ils sont mal effectués, les résultats sont entâchés d'erreurs dont les causes peuvent être assez diverses.

Dans les analyses simplifiées qui ont été présentées plus avant, le barrage est une structure monodimensionnelle où l'on a fait l'hypothèse que la contrainte de cisaillement est constante sur une horizontale. De véritables calculs bidimensionnels montrent que cette condition n'est 
Amont

Aval

Upstream

Dowstream

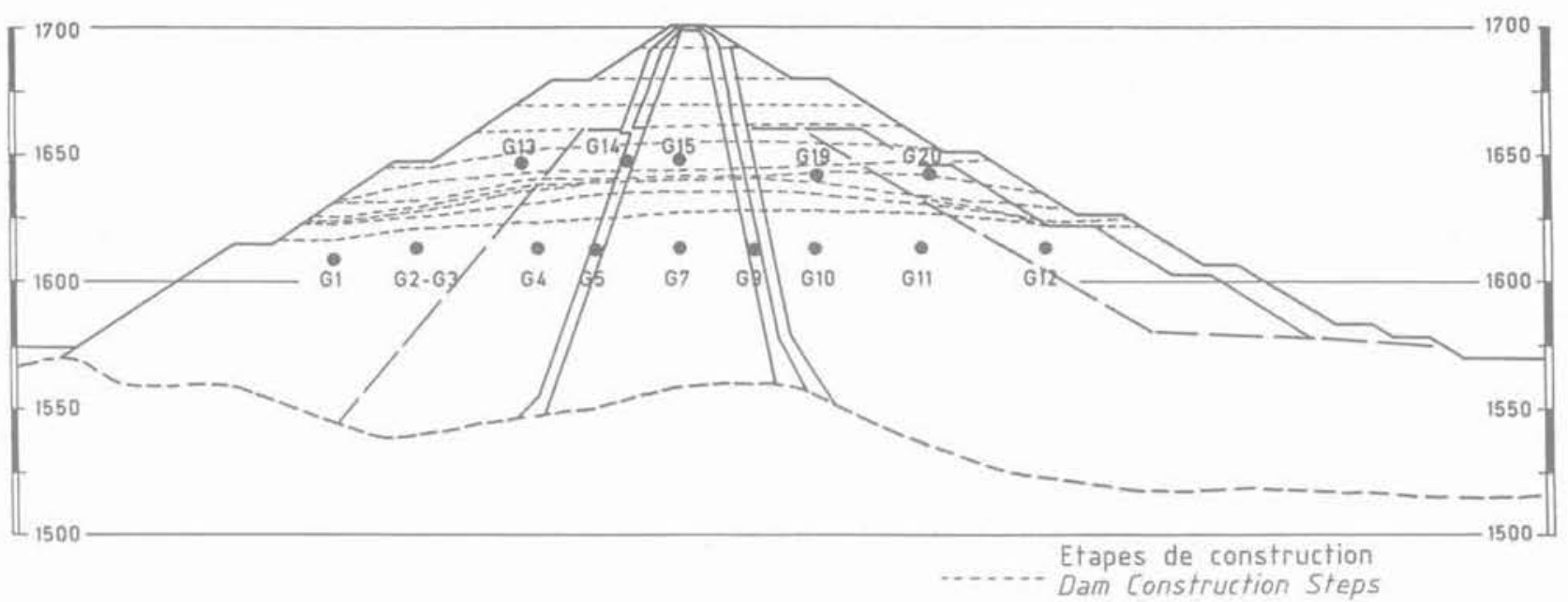

Fig. 2, - Barrage de Grand'Maison.

Implantation du système d'auscultation.

Fig. 2. - Grand'Maison Dam - Monitoring scheme planting.

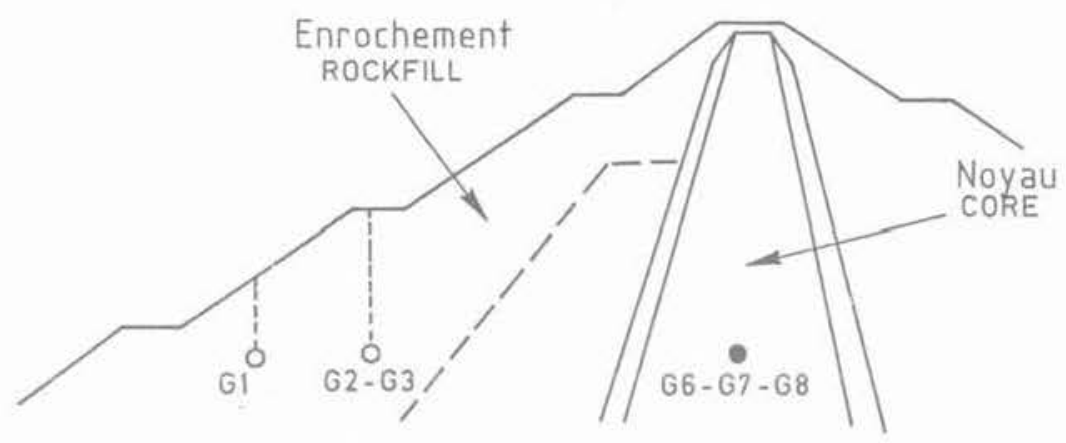

$G\left(\mathrm{MPa}_{\text {moy }}\right)$

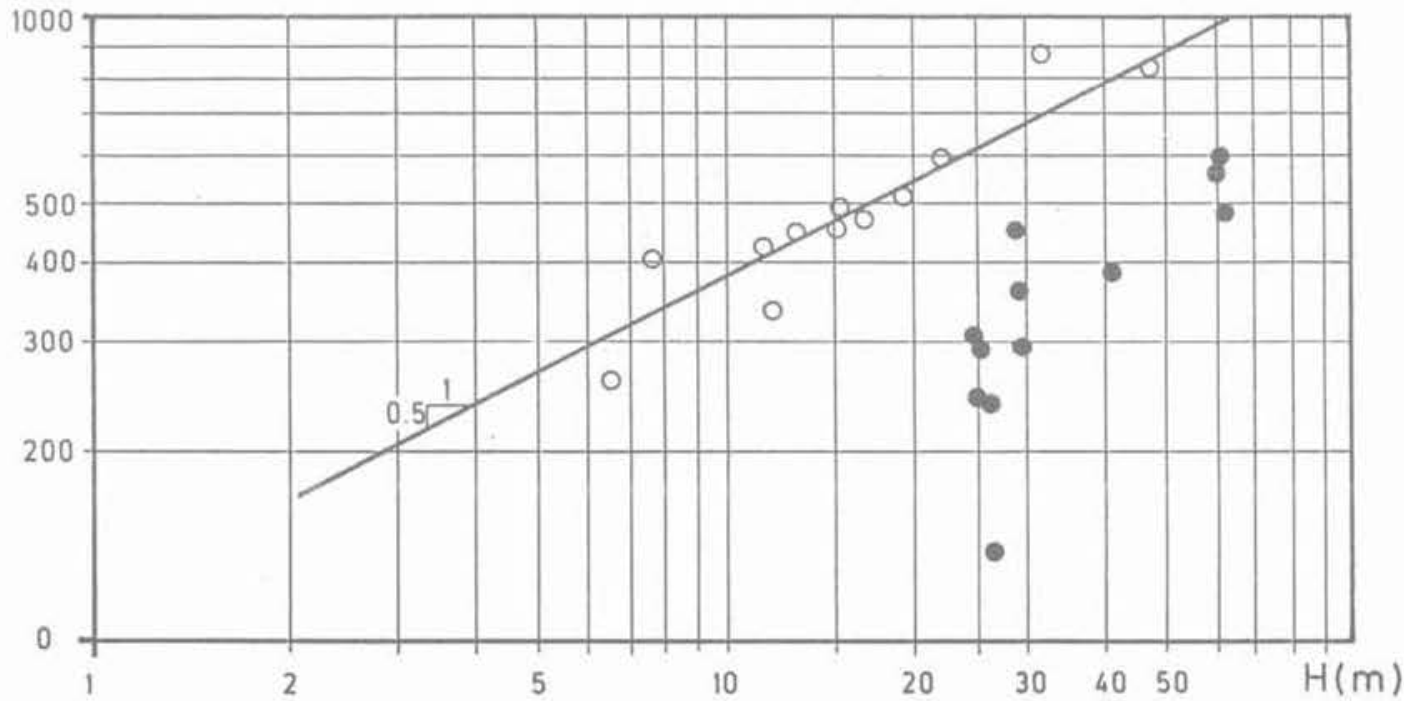

Fig. 3. - Barrage de Grand'Maison.

Evolution des modules de cisaillement

Fig. 3. - Grand'Maison Dam.

avec la hauteur des remblais. 
- Résultat de la simulation à partir de la loi élastoplastique de JC HUJEUX Résult of simulation from JC HUJEUX élastoplastic law

\section{$G / G \max$}

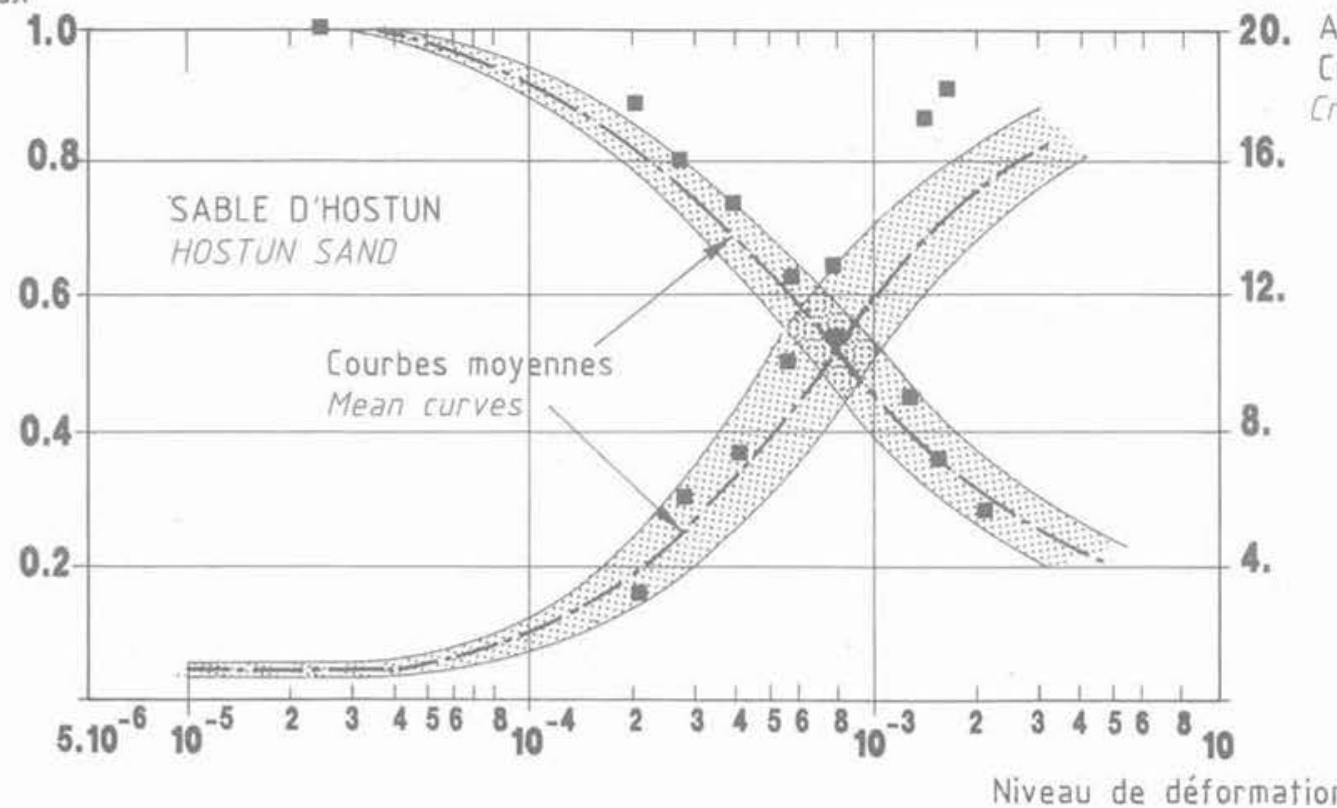

Niveau de déformation
0. Amortissement Critique Critical Damping

Fig. 4. - Modèle équivalent linéaire.

Variation des propriétés visco-élastiques

avec le niveau de déformation de cisaillement. sable d'Hostun (JJ FRY-1987).

Fig. 4. - Linear equivalent model.

Variation of visco-elastio properties with shear deformation. Hostun sand (by JJ FAY-1987).

pas valable dès que l'on s'éloigne de l'axe du barrage, et que par conséquent cette hypothèse est d'autant meilleure que le barrage est plus raide (réf. $3,9,16,18$ ). Pour une étude plus détaillée, la modélisation bi ou tridimensionnelle aux éléments finis sera bien préférable, et c'est la configuration géométrique de l'ouvrage qui devrait guider le choix entre les deux. Pour pouvoir raisonnablement faire l'hypothèse de la déformation plane d'une section du barrage, le rapport $\mathrm{L} / \mathrm{H}$ de la longueur en crête sur la hauteur maximale doit être supérieur à 4 pour une vallée en $U$, et supérieur à 6 pour une vallée en $V$ (réf. $21,23,29$ ). Il faut noter que dans le même temps la sollicitation sismique n'a aucune raison d'agir seulement dans la direction amont-aval, et que par conséquent la réponse sera tridirectionnelle (réf. 27). Quoi qu'il en soit, la méthode des éléments finis permet d'étudier la réponse dynamique de manière plus fine en permettant une modélisation fidèle de la géométrie et de la fondation du barrage. Les caractéristiques des matériaux peuvent être définies au niveau de chaque élément, ce qui permet de réaliser, soit des calculs itératifs avec mise à jour des propriétés en fin de chaque étape pour la méthode équivalente linéaire, soit des calculs non linéaires qui prennent en compte les conditions locales de contraintes et de déformations. Enfin, on obtient les résultats en fonction du temps, qui autorisent d'intéressantes possibilités de post-traitement pour les calculs à la rupture dont il sera question plus loin.
Un dernier point concerne la définition des conditions aux limites aux frontières du modèle. La présence d'un substratum rocheux, ou au contraire d'un demi-espace infini influence de manière notable la réponse dynamique (réf. 5). Sur la figure 6 , on trouve la comparaison des accélérations maximales au cours du temps à la verticale de la crête de barrages identiques, soumis à la même sollicitation sismique, mais qui est imposée à différentes profondeurs du substratum sous le barrage.

\section{CALCULS A LA RUPTURE}

Les calculs à la rupture sont entièrement découplés des analyses dynamiques dont il a été question jusqu'à maintenant. Historiquement on a d'ailleurs commencé par remplacer l'action du séisme par un système supposé équivalent de forces statiques, la nature temporelle de la sollicitation dynamique étant ainsi parfaitement éludée (TERZAGHI, 1950). Un coefficient «pseudo-statique », dont le choix est relativement arbitraire, détermine l'intensité de ce système de force. 

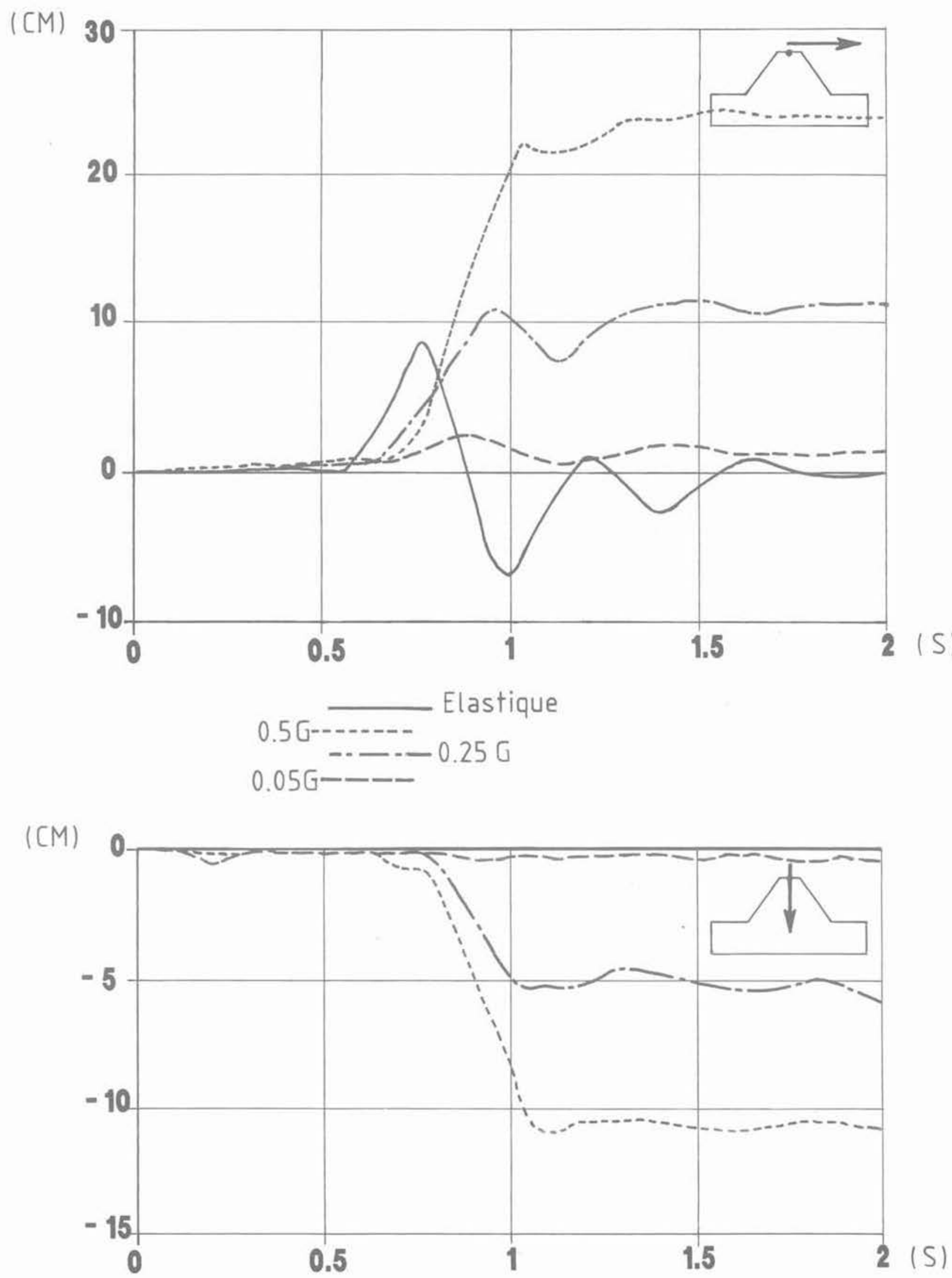

Fig. 5. - Calcul dynamique non linéaire d'un barrage en terre. Déplacement horizontal et vertical en crête.

Fig. 5. - Nonlinear dynamic calculation of an earth dam. Crest vertical and horizontal displacements. 


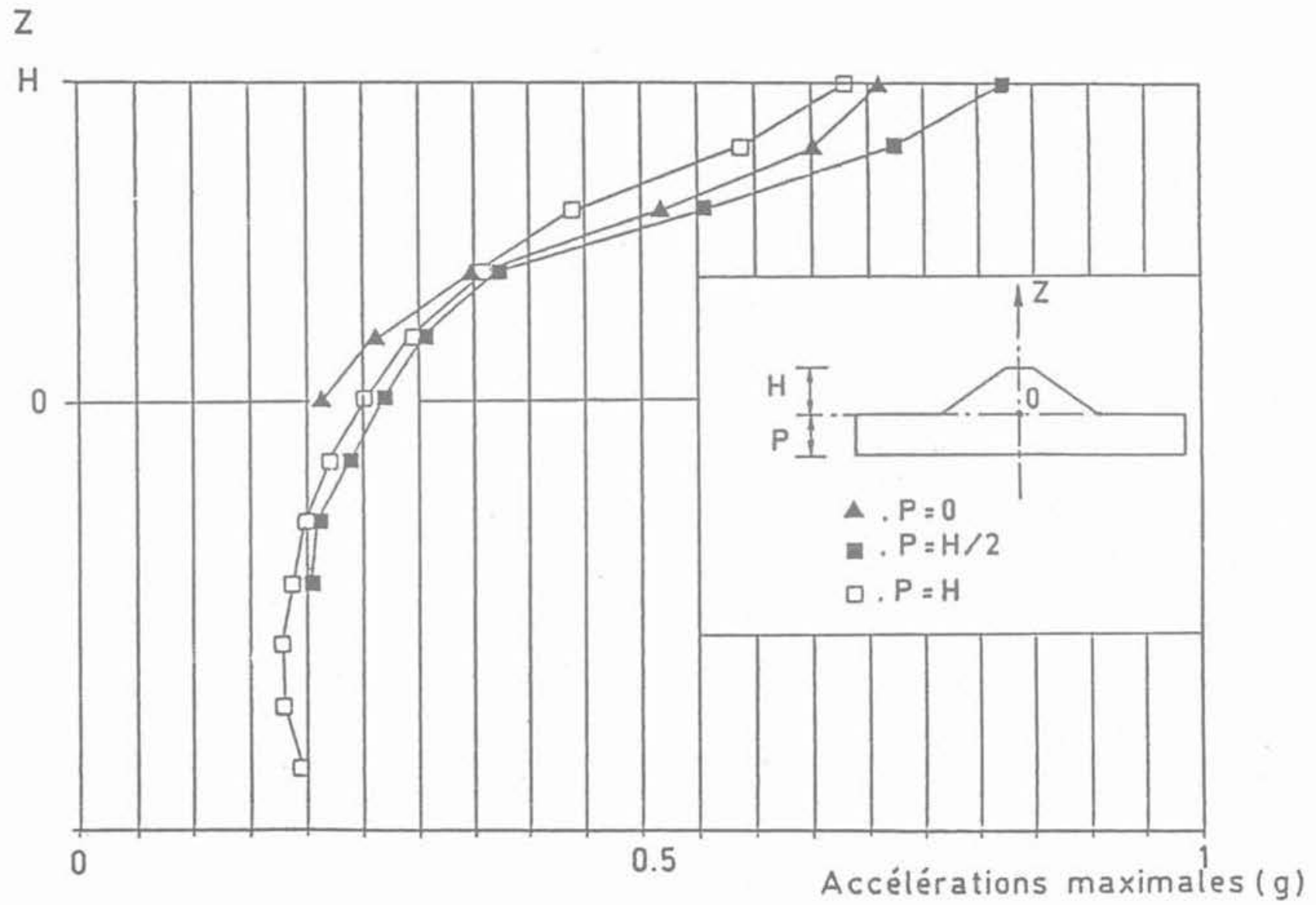

Fig. 6. - Influence des conditions limites sur la réponse dynamique des barrages (réf. 5).

Fig. 6. - Influence of boundary conditions of the dynamic responses of dams (ref. 5).

Tableau 1. - Valeur du coefficient pseudo-statique. Table 1. - Design value of pseudo-static coefficient

\begin{tabular}{|c|c|c|}
\hline \multirow{2}{*}{ Pays } & \multicolumn{2}{|c|}{ Intensité du séisme } \\
\cline { 2 - 3 } & Faible & Fort \\
\cline { 2 - 3 } & 0,05 & 0,15 \\
USA .................. & 0,12 & 0,25 \\
\hline
\end{tabular}

L'analyse de la rupture se base sur la stabilité d'une masse délimitée par une ligne de glissement soumise à un système de forces pseudo-statiques. La méthode que l'on vient de présenter a fait l'objet de nombreux développements qui cherchaient essentiellement à mieux définir le passage du chargement dynamique au système de forces statiques équivalentes (réf. 30). Ce type d'approche doit être réservé aux sols dont on peut assurer que la résistance au cisaillement ne variera pas notablement au cours du séisme, ce qui exclut tous les cas de liquéfaction. D'autre part la définition de la stabilité avec le même critère que pour un chargement statique n'a pas tellement de sens, dans la mesure où un coefficient de sécurité peut parfaitement devenir inférieur à 1 pendant une fraction de seconde sans dommage pour le barrage. Cette idée revient à NEWMARK (1965), qui a proposé d'évaluer la stabilité d'une ligne potentielle de rupture en fonction du déplacement cumulé à la fin du séisme (réf. 26, 15). Le calcul du déplacement se base sur la définition d'une accélération critique qui déclenche le mouvement lorsqu'elle est dépassée. Plutôt que d'évaluer un coefficient sismique moyenné à partir des résultats d'un calcul dynamique, pour l'injecter ensuite dans un calcul de cercle de rupture, il parait plus judicieux de calculer à chaque pas de temps le facteur de sécurité d'une ligne potentielle de rupture à partir des contraintes dynamiques, et des caractéristiques de résistance des matériaux. Cette méthode a été développée dans un calcul en post-traitement de calculs dynamiques (réf, 7). Dès que le moment moteur devient supérieur au moment résistant, le mouvement se déclenche (fig. 7). Les caractéristiques de résistance des matériaux peuvent évoluer en fonction du déplacement de la masse glissante, tel que cela a été préconisé par GOODMAN et SEED (réf. 15, 7).

En ce qui concerne le problème de la liquéfaction, les moyens d'analyse du problème dynamique couplé nous offre un large champ d'application. Jusqu'à présent, le 


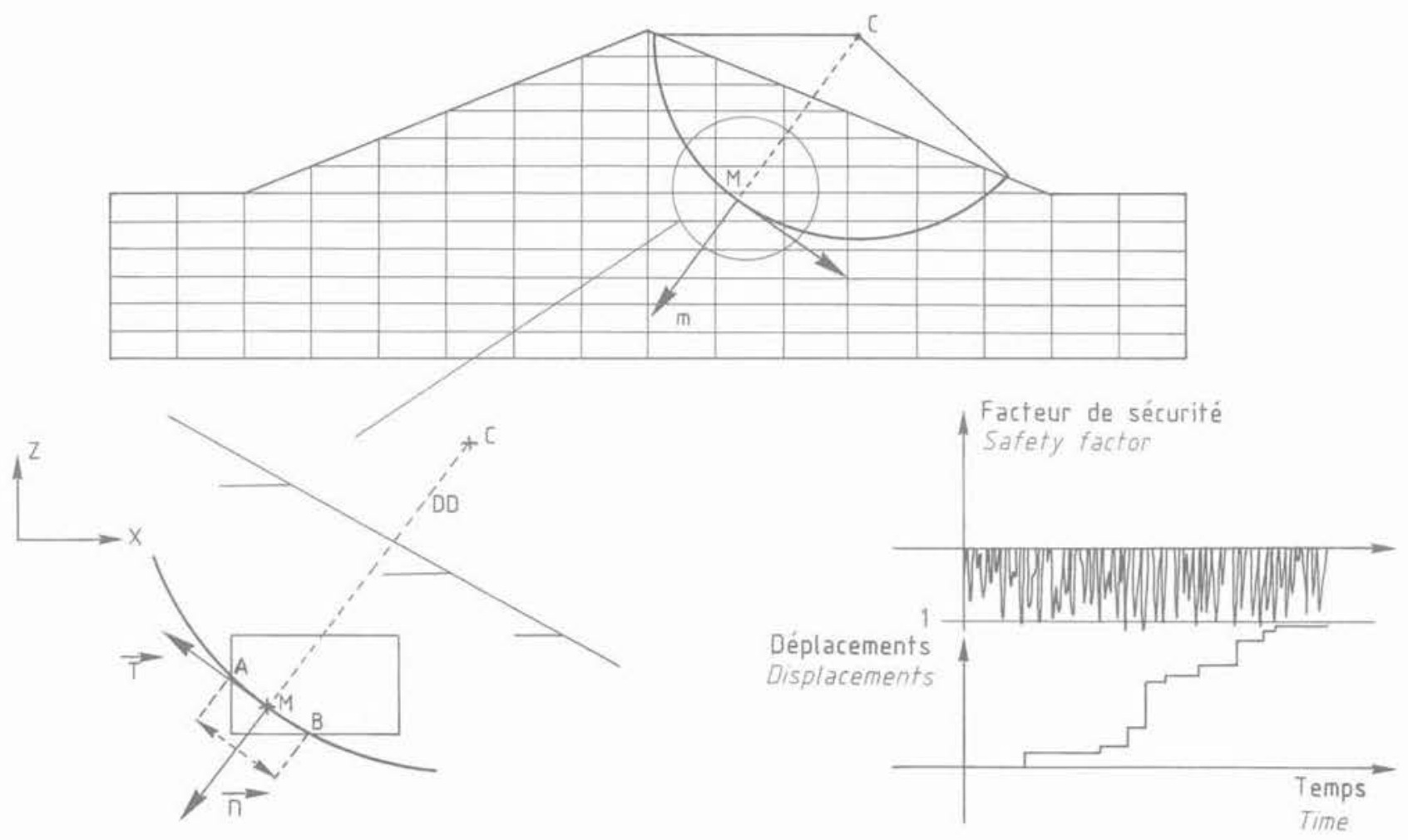

Fig. 7. - Principe du post-traitement par la méthode de Newmark.

Fig. 7. - Postreatment principle of Newmark method.

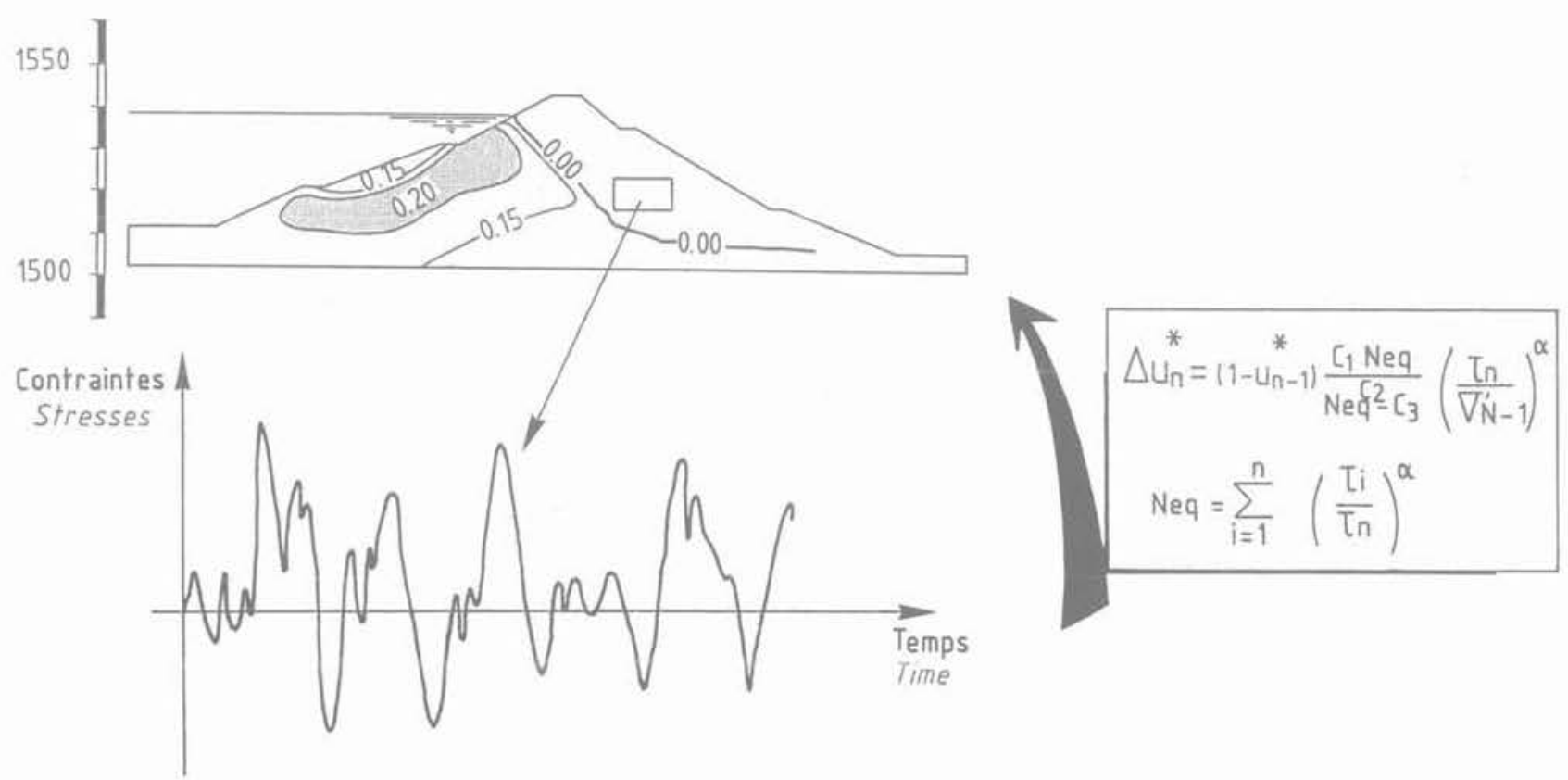

Fig. 8. - Principe du calcul

de la surpression interstitielle (MPa)

à la fin du séisme dans le barrage de Matemale (réf. 8).

Fig. 8. - Principle of pore water pressure generation

inside Matemale dam. (ref, 8). 
problème a été traité de façon pragmatique, soit sur la base de la méthode proposée par SEED et LEE (réf. 32), soit à partir de lois calées sur des essais de laboratoire, et qui permettent de relier la génération de pression interstitielle au chemin de contrainte totale que lon a calculé de manière indépendante (réf. 6). Le principe d'intégration proposé par SHERIF (réf. 33), a été utilisé en post-traitement de calculs dynamiques pour la détermination d'un champ de surpressions interstitielles en fin de séisme (réf. 8). Un exemple de résultat est indiqué à la figure 8. La loi qui est proposée par SHERIF intègre la variabilité du signal sismique, ainsi que l'influence du nombre et de l'amplitude des cycles. Cependant les paramètres de la loi sont déterminés à partir d'essais triaxiaux normalement consolidés, ce qui conduit à de fortes générations de pressions interstitielles. Une amélioration consiste à pondérer ces résultats en fonction de la distance du point représentatif des contraintes à la droite de rupture (réf. 6). Ces approches sont intéressantes, mais demandent un important support expérimental pour être validées.

\section{CONCLUSIONS}

La réponse dynamique des barrages en terre offre encore de nombreuses voies de recherche, en particulier dans le domaine de la dynamique des sols. Les moyens d'analyse sont aujourd'hui très complets, un effort dans le sens de l'allègement des calculs devrait faciliter l'utilisation des lois non linéaires. Les analyses équivalentes linéaires ne sont pas pour autant à rejeter puisqu'elles représentent correctement le comportement des sols dans une gamme de déformation de $10^{-6}$ à $10^{-3}$. Les analyses en contraintes totales offrent des possibilités de post-traitements faciles à mettre en œuvre, et dont les résultats sont tout à fait intéressants dans le domaine du calcul à la rupture, ou dans lévolution des effets irréversibles du séisme : tassements, génération de pressions interstitielles. Enfin, le plus gros effort doit être dirigé vers la meilleure connaissance du domaine de validité, ainsi que vers la détermination pratique, en laboratoire ou in situ, des paramètres de chaque modèle de comportement.

\section{BIBLIOGRAPHIE}

1. ABDEL-GHAFFAR A.M., ELGAMAL A.M. (1987), Elasto-plastic - Seismic response of 3-D earth dams: theory. Journal of Geotechnical Engineering, ASCE, vol. $113, n^{\circ} 11$, novembre 1987 , p. 1293.

2. ABDEL-GHAFFAR A.M., KOH A.S. (1981), Lon. gitudinal vibration engineering and structural dynamics. ASCE, vol. 9, 1981, pp. 279-305.

3. AMBRASEYS N.M. (1960), Consideration on the vibrational behaviour of earth dams. Bull. $n^{\circ} 52$, Disaster Prevention Research Inst., Kyoto Univ., Kyoto (Japon).

4. AZIMI, AVRIL T. (1987), Mesures in situ des caractéristiques dynamiques des matériaux du barrage de Grand'Maison. Journées EDF : * Calcul dynamique des barrages », juin 1987.
5. AVRIL T. (1987), Analyse par plusieurs méthodes de l'interaction dynamique sol-structure dans un barrage. Coll. «Utilisation de la méthode des éléments finis dans les projets de géotechnique ", Paris, 1987.

6. AVRIL T. (1987), Programme Liquef. Note technique EDF, juin 1987 .

7. AVRIL T. (1986), Programme Newmark. Note technique EDF, avril 1986.

8. AVRIL T., FRY J.J. (1987), Evaluation of resilient and permanent deformations as well as pore pressure generation of earth and rock fill materials under cyclic loading. International symposium on earthquakes and dams, 20th May 1987. Beijing (China).

9. CHOPRA A.K. (1967), Earthquake response of earth dams. Journal of soil mechanics and foundations division, ASCE, vol, $n^{\circ} 3, n^{\circ}$ SM2, $p p$. 65-81.

10. DUPAS J.M., PECKER A., BOZETTO P., FRY J.J. (1986), A $300 \mathrm{~m}$ diameter triaxial with a double measuring device. Symposium of advanced triaxial testing of soil and rock, ASTM, juin 1986. Louisville (Kentuky).

11. ELGAMAL A.M., ABDEL-GHAFFAR A.M. (1987), Elasto-plastic seismic response of 3.D earth dams : application. Journal of Geotechnical Engineering, ASCE, vol. $113, \mathrm{n}^{\circ} 11$, novembre 1987 , pp. 1309-1325.

12. ELGAMAL A.W.M., ABDEL-GHAFFAR A.M., PREVOST J.H. (1984), Elasto-plastic earthquake shear response of 1-D non homogeneous earth dam models. Journal of Geotechnical Engineering, ASCE.

13. ELGAMAL A.W.M., ABDEL-GHAFFAR A.M., PREVOST J.H. (1987 a), 2-D elasto-plastic seismic shear response of earth dams : $L$. theory. J. Engrg. Mech., ASCE, 113 (5), pp. 689-701.

14. ELGAMAL A.M., ABDEL-GHAFFAR A.M., PREVOST J.H. (1987 b), 2.D elasto-plastic seismic shear response of earth dams : II. applications. J. Engrg. Mech., ASCE, 113 (5), pp. 701-719.

15. GOODMAN R.E., SEED H.B. (1966), Earthquake induced displacements in sand embankments. Journal of the soil mechanics and foundation division, ASCE, vol. 92, n SM2, pp. 125-146.

16. HATANAKA M. (1955), Fundamental considerations on the earthquake resistant properties of the earth dam. Bull. $n^{\circ} 11$, Disaster Prevention Research Inst., Kyoto (Japon).

17. HERZOG A.M. (1985), Closed formulae for induced deformations in dams. Journal of water power an dam construction, janvier 1985, pp. 33-34.

18. ISHIZAKI H., HATAKEYAMA N. (1960), Consideration on the vibrational behaviour of earth dams. Bull $n^{\circ} 52$, Disaster Prevention Research Inst., Kyoto Univ., Kyoto (Japon).

19. LAGINHA SERAFIM J. (1987), Effects caused by earthquakes on dams. Mai 1987, 55th Execution Meeting of ICOLD, Beijing.

20. LUONG M.-P. (1986), Mesure des propriétés dyna. miques des sols. Revue Française de Géotechnique, $n^{\circ} 37,4^{e}$ trimestre 1986. 
21. MAKDISI F.I., KAGAWA T., SEED H.B. (1982), Seismic response of earth dams in triangular canyons. Journal of Geotechnical Engineering Division, ASCE, vol. 108, $n^{\circ} 10$, pp. 1328-1338.

22. MAKDISI F.I., SEED H.B. (1978), Simplified procedure for estimating dam and embankment earthquake induced deformations. Journal of the Geotechnical Engineering Division, vol. 104, n GT7, juillet 1978 .

23. MEJIA L.H., SEED H.B. (1983), Comparison of 2-D and 3-D dynamic analysis of earth dams. Journal of Geotechnical Engineering, ASCE, vol. 109, $\mathrm{n}^{\circ}$ 11, pp. 1383-1398.

24. MODARESSI $H_{\text {, }}$ AUBRY D., MOUROUX $P$. (1986), Wave propagation in a saturated porous media. Proc. of 8th Europear Conference on earthquake engineering, Analytical method in soil dynamics, Lisbonne 1986.

25. MONONOBE N., TAKATA A., MATUMURA M. (1936), Seismic stability of an earth dam. Trans.. vol $n^{\circ} 4,2$ d Congress on Large Dams, Washington DC.

26. NEWMARK N.M. (1965), Effects of earthquakes on dams and embankments. 5th Rankine Lecture. Géotechnique, vol. $15, \mathrm{n}^{\circ} 2$, pp. 139-160.

27. OKAMOTO E.S., TAMURA C., KATO K. et al. (1967), Dynamic behaviour of earth dam during earthquake. Proc. 9th, Congrès des Grands Barrages, Q 35, R 6, pp. 111-123.

28. PANDE G.N., PIETRUSZCKAK S. (1986), A cri. tical look at constitutive models for soils. Geome- canical Modeling in Engineering Practice, Balkema, 1986.

29. PREVOST J.H., ABDEL-GHAFFAR A.M., LACY S.J. (1985 a), Nonlinear dynamic analysis of an earth dam : a comparative study. Journal of Geotechnical Engineering Division, ASCE, vol. 111, $\mathrm{n}^{\circ} 7$, pp. 882-898.

30. SEED H.B., MARTIN J.R. (1966), The seismic coefficient in earth dam regions. Journal of the Soil Mechanics and foundation division, ASCE, vol. 92. $n^{\circ}$ SM3, mai 1966, pp. 25-58.

31. SEED H.B., MAKDISI 1.I., DE ALBA P. (1980), The performance of earth dam during earthquakes. Water Power and Dam construction, vol. $32, n^{\circ} 8$, August 1980, pp. 17-27.

32. SEED H.B., IDRISS I.M., LEE K.L. (1971), Dynamic analysis of the slide in the lower San Fernando dam during the earthquake of February 9, 1971. Journal of the Geotechnical Engineering Division, ASCE, vol. 101, n० GT9, septembre 1975, pp. 889.911.

33. SHERIF M.A., ISHIBASHI I, et TSUSHIYA C. (1978). Pore pressure prediction during earthquake loadings. Journal of Soils and Foundations, Japanese Society of Soil Mechanic, vol. 18, $\mathrm{n}^{\circ} 4$, décembre 1978.

34. TARDIEU (1983), Méthodes simplifiées pour le prédimensionnement d'un barrage fondé sur rocher soumis à un séisme. Colloque technique du Comité Français des Grands Barrages, Paris, mars 1983.

\section{BRGM}

\section{Ingénierie Géotechnique}

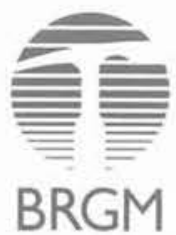

BP 6009 - 45060 ORLEANS CEDEX 2 - FRANCE

TEL. (33) 38.64.37.20

TELECOPIEUR : 3864.36 .43 